Uses and abuses of EIDORS: An extensible software base for EIT

Adler, Andy and Lionheart, William R.B.

2006

MIMS EPrint: 2008.25

Manchester Institute for Mathematical Sciences

School of Mathematics

The University of Manchester

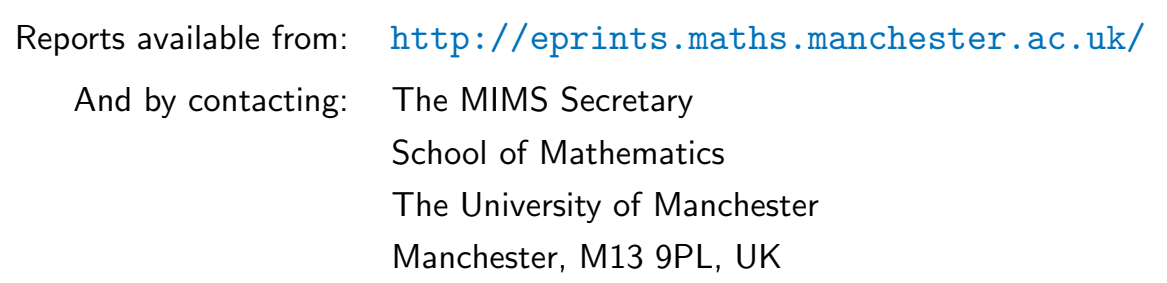

ISSN 1749-9097 


\title{
Uses and abuses of EIDORS: An extensible software base for EIT
}

\author{
Andy Adler ${ }^{1}$, William R. B. Lionheart ${ }^{2}$ \\ ${ }^{1}$ School of Information Technology and Engineering, University of Ottawa, Canada \\ 2 School of Mathematics, University of Manchester, U.K. \\ E-mail: adler@site.u0ttawa.ca, bill.lionheart@manchester.ac.uk
}

\begin{abstract}
.
EIDORS is an open source software suite for image reconstruction in electrical impedance tomography and diffuse optical tomography, designed to facilitate collaboration, testing and new research in these fields. This paper describes recent work to redesign the software structure in order to simplify its use and provide a uniform interface, permitting easier modification and customization. We describe the key features of this software, followed by examples of its use. One general issue with inverse problem software is the difficulty of correctly implementing algorithms, and the consequent ease with which subtle numerical bugs can be inadvertently introduced. EIDORS helps with this issue, by allowing sharing and reuse of well documented and debugged software. On the other hand, since EIDORS is designed to facilitate use by non-specialists, its use may inadvertently result in such numerical errors. In order to address this issue, we develop a list of ways in which such errors with inverse problems (which we refer to as "cheats") may occur. Our hope is that such an overview may assist authors of software to avoid such implementation issues.
\end{abstract}

Keywords: Electrical Impedance Tomography, Open Source, Inverse Problems

\section{Introduction}

EIDORS (Electrical Impedance and Diffuse Optical tomography Reconstruction Software) is a software suite for image reconstruction in electrical impedance tomography (EIT) and diffuse optical tomography (DOT). Its goal is to provide a freely distributable and modifiable software for image reconstruction of electrical or diffuse optical data. Such software facilitates research and development in these fields by providing a reference implementation against which new developments can be compared, and by providing a functioning software base from which new ideas may be built and tested. Making the source code available also facilitates scrutiny of algorithms and their implementation by other researchers. The original EIDORS (version 1) software [17] is based on software from the thesis of Vaukhonen [16]. It implemented a MATLAB package for two-dimensional mesh generation, solving of the forward problem and reconstruction and display of the images. In order to provide capability to solve 3D reconstruction models, a new project, EIDORS3D (version 2), was begun [11], based on 
the software developed for the thesis of Polydorides [12] The EIDORS software packages shared the same numerical and algorithmic foundations, but shared very little software code. Each software package modelled the medium under investigation using a simplex based finite element representation, and images were reconstructed using regularized inverse techniques.

In the three years since the publication of EIDORS3D, several patterns of use have been noted. Researchers typically download the software, run the provided demonstration examples, and make modifications in the demonstration examples and the software internals to meet their needs. Because of the lack of a modular software structure of EIDORS3D, changes tended to be made into the code itself. This resulted in duplicated code which could not easily be refactored in order to be contributed back to EIDORS. Additionally, recent work has begun to move away from basic reconstruction algorithms, focussing on such issues as mesh generation, electrode modelling, visualization and electrode error detection. Such research would be facilitated by using modular components which could be "plugged" into a selection of reconstruction algorithms.

To address these issues, the EIDORS software has been completely restructured with the goal of providing an extensible software base designed to support community use, modification and contribution. These modifications have been released as EIDORS version 3 (currently at release 3.1 ), which incorporates the following features:

- Multiple algorithm support: EIDORS V.3 has been redesigned to allow flexibility of using multiple algorithms (or parts of algorithms). This version provides access to the algorithms of [1], [3], [11], [14] and [17]. We feel that this capability is becoming more important with a trend toward meta-algorithms in EIT, such as algorithms for detection of electrode errors [2].

- Generalized model formats: One limitation of previous versions of the EIDORS software is that they were designed around specific electrode configurations and stimulation and measurements patterns. While it was possible to use the software for more general configurations, this was a fairly daunting task. In order to support the wide variety of EIT measurements and algorithms, EIDORS now provides a general EIT model format (iethe $\mathrm{f}$ wd_model structure). Additionally, several utility functions are provided to create common electrode and stimulation configurations. This format specifies the electrode positions, contact impedances, and stimulation and measurement patterns, and all supported algorithms are able to reconstruct images based on data provided in these formats.

- Interface software for common EIT systems: functions are provided (in the interface directory) to load from a variety of EIT hardware storage formats into the EIT data format. Currently, EIDORS will detect when data do not match the specified protocol in the $\mathrm{fwd}$ model, but will not (yet) automatically convert the data. At this point it has only been possible for the developers to offer support for EIT hardware that they have access to. We hope that EIDORS will attract contributions from 
software developers who have access to other hardware systems.

- Usage examples: It is observed that researchers typically base new software on demonstration examples. To facilitate this, several simple and more complex usage examples are provided for image reconstruction in two and three dimensions using various image reconstruction algorithms and combinations of algorithms.

- Test suite: Software is intrinsically difficult to test. While little work has been done specifically on testing numerical software for inverse problems, we believe that such tests are even more difficult. EIDORS has begun to implement a series of regression test scripts (in the tests directory) to allow automatic testing of code modifications. For example the function calc_jacobian_test.m validates a function to calculate the Jacobian against an approximation of the Jacobian using the perturbation method.

- Open-source license: EIDORS is licensed under the GNU General Public License [9]. Users are free to use, modify, and distribute their modifications. All modifications must include the source code, or instructions on how to obtain it. EIDORS may be used in a commercial product, as long as the source code for EIDORS and all modifications to it are made available. In addition, by making EIDORS available in this way, we would like to encourage other authors to publish their scientific software, in order to make results more easily verifiable and repeatable.

- Sourceforge hosting: In order to allow collaborative development, EIDORS is hosted by sourceforge.net available at http://eidors.org or http://eidors3d.sf.net. Software is available for download as packaged released versions (version 3.1 was released on 24 Jan 2006, and contains the features described in this paper), or the latest developments may be downloaded from the Concurrent Versions System (CVS) [4]. Sourceforge hosting allows for collaborative development for group members, while permitting read-only access to everyone. In order to become a member of the developer group, new contributors should contact the authors. One concern with a distributed software project is the possibility of conflict due to disparate authors working on the same module. Version control software such as CVS is widely understood to facilitate collaborative development, and manage software version conflicts [4].

- Language independence: (Octave and Matlab) EIDORS was originally written for Matlab. However, the eventual goal is to support multiple mathematical software packages. Some progress has been achieved, and EIDORS version 3 works with Octave [8], although some advanced graphics functions still function only with Matlab. The current version of EIDORS works with Octave (version $\geq 2.9 .4$ ) and Matlab (version $\geq 6.0$ ). Support for Octave was motivated by two goals: first, octave provides a free software platform which can encourage the development of embedded and commercial applications of EIT which are currently limited by the cost of Matlab, and secondly, Octave provides an open source platform to match the open source nature of EIDORS. 
- Pluggable code base: In order to facilitate user modifications, EIDORS has been designed to provide some of the benefits of object-oriented (OO) software [10]: abstraction, encapsulation, polymophism and inheritance. We have decided not to use the OO framework provided by Matlab, because in our experience it is somewhat cumbersome, and may be intimidating to many mathematicians and engineers who do not regularly write OO software. Another issue is the unfortunate reputation of the Matlab language for undergoing dramatic modifications to new language features in the first few versions. For these reason, EIDORS has been designed using a "pluggable" software approach. This design uses function pointers to allow adding new modules and controlling which parts of functions are executed. EIDORS is thus able to offer the OO features of packaging and polymophism, while not encapsulation or inheritance. A detailed description of this capability is provided in the next section. While EIDORS does not yet provide algorithms for DOT, this aspect of the software architecture allows integration of such algorithms with the advanced inverse solvers provided.

- Automatic matrix caching: In order to increase performance of image reconstruction software, it is important to save and reuse values of computationally expensive variables, such as the Jacobian and image priors. Such caching complicates the software implementation, and potentially leads to errors. In order to simplify design, EIDORS offers the ability to automatically detect when a calculation requests a value previously calculated, and will automatically retrieve that previous value. EIDORS extracts caching to a separate module eidors_obj, in a way that will generally happen transparently for the programmer. This capability helps software based on EIDORS to be more clear and easier to decompose into functional modules.

- Enhanced Finite Element Modelling and Graphical output: Image reconstruction, especially in 3D, requires functions to show high quality graphical representations of the images. EIDORS provides several functions for image presentation using the Matlab graphics features, as well as functions to display images using the VTK visualization program [13], after exporting the data to a vtk file. show_fem.m displays a three dimensional model of the finite element mesh and conductivity changes, while show_slices.m allows generation of images of arbitrary two dimensional slices through a volume. Examples of these functions are shown in Figs. 5 and 6. All EIDORS graphics functions now use a single colour mapping function calc_colours.m, which allows global modification of all image colouring using a global variable eidors_colours. For modelling, the create_tank_mesh_ng.m function is provided to generate a finite element mesh of a cylindrical tank with circular of rectangular electrodes using the netgen [15] mesh generator.

We hope that by providing a structure for community collaboration in EIT algorithms, we can produce robust, reliable and fairly portable software which draws on our collective 
expertise and facilitates innovations in the field.

\section{Software Architecture}

This section describes the structure of EIDORS objects and their relationship to the underlying finite element models and numerical functions.

\subsection{Object Structure}

EIDORS software consists of four primary objects: data, image, fwd_model, inv model. Each object is represented by a structure. All objects have the properties of a name and type. The name is arbitrary; it is displayed by the graphics functions, and could also be useful to distinguish objects in a user specified function. The type is used to identify the object type (ie data, image etc.).

\subsection{1. fwd_model :}

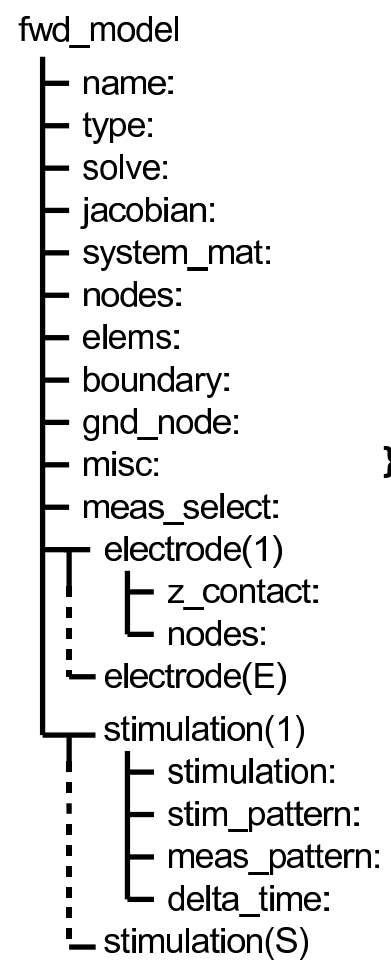

'NP 3D zigzag mdl'

'fwd_model' eg. $n p \_f w d \_s o l v e$ eg. $n p \_c a l c$ jacobian eg. np_calc_system_mat [Matrix] $V \times \bar{D}$ [Matrix] $N \times(D+1)$ [Matrix] $B \times D$ eg. 252 \} node index \} optional data for algs [Matrix] $E^{2 \times 1}$

eg. 100 \} ohms

eg. $[152,153,215,216]$

eg. ' $m A^{\prime}$

[Matrix] Ex1

[Matrix] $E \times M_{i}$ eg. 0.0 \} optional (sec.)

Figure 1. The structure of the EIDORS fwd_model object.

The most complex EIDORS object is the fwd_model, which is designed to represent the finite element model (FEM), electrode positions and properties, and stimulation patterns, as well as the pointers to functions to solve the forward problem on this model. Fig. 1 illustrates the structure of a fwd_model.

The FEM is described by the fields nodes $(V \times D)$, elems $(N \times(D+1))$, and boundary $(B \times D)$, where $V$ is the number of vertices, $N$ is the number of simplices 
(and also the number of unknown conductivities to be solved by the inverse solution), $B$ the number of simplices with a face on the boundary, and $D$ the model dimension ( $D=2$ for $2 \mathrm{D}$ and $D=3$ for $3 \mathrm{D}$ ). The gnd node is the vertex number attached to ground.

The electrodes are defined by a vector $(E \times 1)$ of electrode fields. Each of $E$ electrode objects has fields $z_{-}$contact (scalar) and nodes (vector) which represent the (possibly complex) contact impedance and vertices to which that electrode is connected. A point electrode would have a single element nodes field with $z_{-}$contact $=0$ while an electrode with a complete electrode model would have multiple vertices specified in nodes and $z_{-}$contact $\geq 0$. Note that EIDORS does not require the electrode model to be the same for all electrodes in a fwd_model.

Using these electrodes, a sequence of $S$ stimulation patterns are applied and measurements performed in order to generate a frame of data. Stimulation patterns are defined by a vector $(S \times 1)$ of stimulation fields. Each stimulation object has fields stimulation, stim_pattern, and meas_pattern. The stimulation is the quantity stimulated into the electrodes. Typically, EIT systems inject current (represented by "mA"), but the quantity could be voltage (or luminous intensity in an optical tomography system). Currently, EIDORS only accepts current stimulations. stim_pattern is a vector $(E \times 1)$ of the stimulation quantity applied to each electrode during that stimulation pattern. Each stimulation object also has an optional delta_time field representing the time increment between measurements at this stimulation and the beginning of the measurement frame. Such data may be used to perform Kalman filtering, for example. meas_pattern is a (sparse) matrix $\left(E \times M_{i}\right)$ representing the $M_{i}$ measurement patterns for stimulation $i$. Each column of this matrix represents the amplification of the signal at each electrode for a single measurement pattern. For example if measurement $k=2$ is the difference signal between electrodes 4 and 5, then meas_pattern ${ }_{j, k}$ is 1 for $j=4,-1$ for $j=5$ and zero for other values of $j$ when $k=2$. EIDORS does not require that the number of measurement patterns be equal for each stimulation pattern. The total number of measurements per frame is $M=\sum_{i=0}^{S} M_{i}$

For many EIT systems, an adjacent stimulation pattern is used with no measurement taken at current stimulation electrodes, giving $M=E \times(E-3)$. For a 16 electrode system, this gives 208 measurements (or, considering reciprocity, 104 independent measurements). One practical consideration is that many EIT systems store data as a matrix of size $\left(E^{2} \times F\right)$ where $F$ is the number of data frames. In this case $E^{2}=256$ of which $3 \times E=48$ measurements in each frame yield zero. In order to allow easy use of EIDORS with such systems, the optional field meas_select $\left(E^{2} \times 1\right)$ is defined for the $\mathrm{fwd}$ model. This field contains a 1 in each position corresponding to a used measurement pattern in the frame (thus meas_select will have $M$ ones).

EIDORS provides several utility functions to define the fields of the fwd model for common patterns, such as the mk_circ_tank.m, mk_stim_patterns.m and mk_common_model.m functions. These functions allow easy definition of circular and 
cylindrical FEM models with rings of electrodes and adjacent stimulation protocols.

The fwd_model contains three function pointers to allow solution of the forward problem, solve, jacobian and system mat. Each field contains the function name (as a string) or a function pointer to calculate these quantities. In each case, these quantities are solved using the utility functions fwd_solve(), calc_jacobian() and calc_system_mat(). For example, given a fwd_model object fmdl, we may calculate the system matrix, Smat, using:

$$
\text { Smat }=\text { calc_system_mat }(\text { fmdl }) \text {; }
$$

This code will call the appropriate function and also manage the caching of the computed result. In this case, if a system matrix has previously been computed for a $f$ wd_model object with the same values as $f m d l$, then the previous result will be returned, without the computation function being called.

2.1.2. data An EIDORS data object represents a frame of measurement or simulated data. The structure of the data objects is shown in Fig. 2. The required fields are the actual frame data, meas, and the acquisition time, time, in seconds after the epoch. In a particular application time may be defined with respect to another start point, such as the start of the experiment, or may be set to 0 or -1 for unknown times or simulated data.
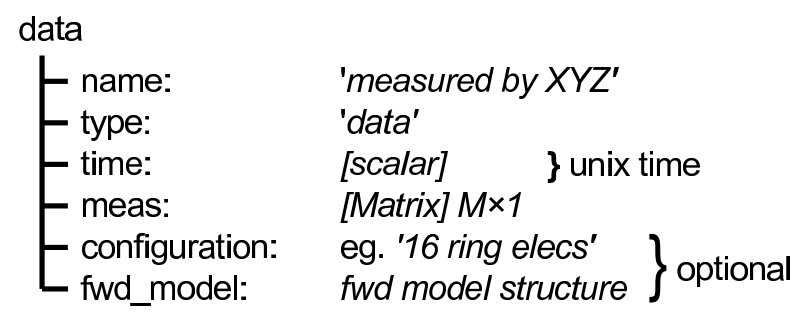

Figure 2. The structure of the EIDORS data object.

The meas field is a $M \times 1$ matrix where $M$ is the number of measurements in each data frame (the sum of number of measurements for each stimulation pattern). The data in meas is ordered such that the measurements for stimulation(1) are first.

Data may be loaded into EIDORS using the eidors_readdata() function, which provides an interface to the storage formats used by some EIT equipment manufacturers.

The data object may also contain two options fields, configuration and $f w d$ model. Specification of $f w d$ model allows EIDORS to validate that the data are being interpreted correctly, and being reconstructed using the correct model. The configuration is a user specified string with a similar function; software may assign a value to this field in order to distinguish data objects.

2.1.3. inv_model The inv model object groups information necessary to allow reconstruction of images. Two basic types of reconstruction are distinguished based on 


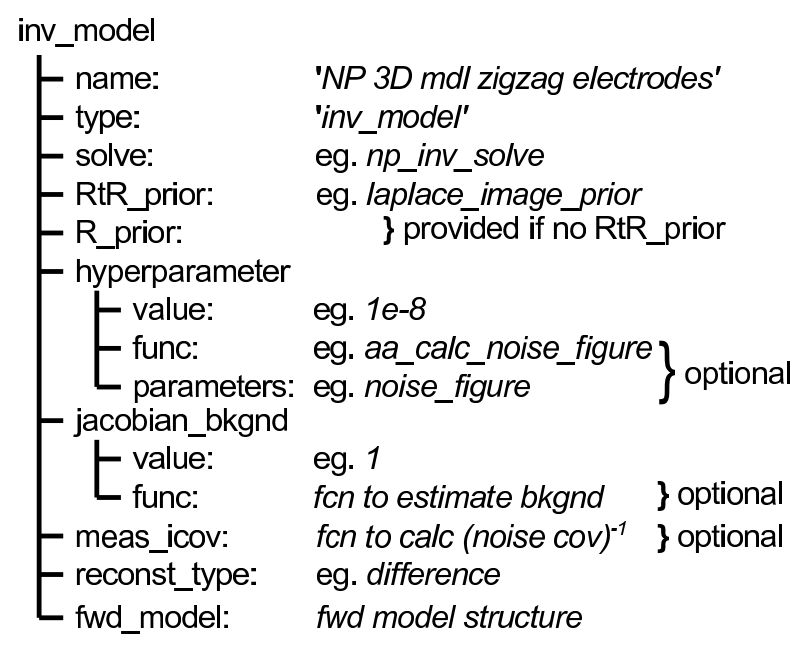

Figure 3. The structure of the EIDORS inv_model object.

the reconst_type field, "difference" (which calculates an image based on the difference between two data objects) and "static" (which calculates an image based on a single data object.

The pointer to the solver function is stored as a string or function pointer in the solve field. The provided functions are based on regularized image reconstruction algorithms, and require an image prior and a choice of hyperparameter. The latter is specified in the hyperparameter field. For simple cases, a scalar value is provided in the value field. However, for more complex cases, a hyperparameter selection strategy is specified as a function in the func field. One example is the noise figure strategy of [1]. This strategy may be specified by setting hyperparameter.func='aa_calc_noise_figure' and hyperparameter .noise_figure=Value.

Image priors are commonly used in two ways, using either a regularization term of $\|\lambda \mathbf{R x}\|$ or $\|\lambda \mathbf{x}\|_{R}$, where $\mathbf{x}$ is the vector of image element values. In the most common case, a quadratic norm is used, and these regularization terms may be expressed as $\lambda^{2} \mathbf{x}^{t} \mathbf{R} \mathbf{x}$ or $\lambda^{2} \mathbf{x}^{t} \mathbf{R}^{t} \mathbf{R} \mathbf{x}$, respectively. One implementation concern is to make it clear to the user which type of image prior is required. EIDORS addresses this by defining two different functions to calculate image priors, R_prior and RtR_prior. A user may provide a value for either or both. When the algorithm specified in the solve field is called, it will request either an R_prior or RtR_prior. If the RtR_prior is required, but

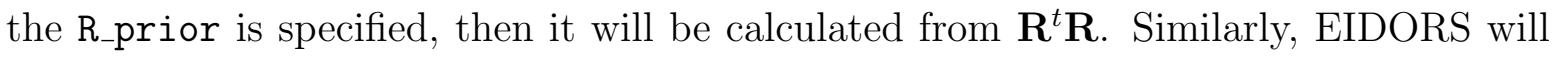
attempt to calculate $\mathbf{R}$ from the Cholesky factorization of the RtR_prior, if required. Parameters to an image prior function are specified in a field, using the name of the image prior function, added to the inv model object.

2.1.4. image The EIDORS image object expresses the reconstructed or simulated conductivity values. The field elem_data $(N \times 1)$ is the value of each of the image 
elements in the finite element model (in the field $\mathrm{fwd}$ model).

For example, given an inv_model object imdl, we may express image reconstruction by (assuming difference EIT, and data objects data1 and data2):

img = inv_solve( imdl, data1, data2 );

Similarly, in order to simulate data object datasim from a simulation image imgsim, we may write:

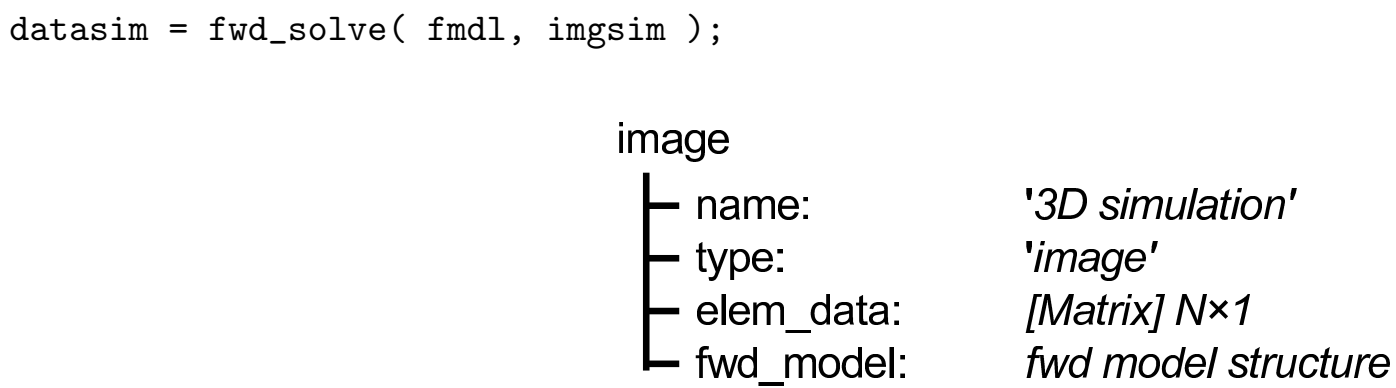

Figure 4. The structure of the EIDORS image object.

\subsection{Software Example: Calculation of the Jacobian}

In order to illustrate the usage of EIDORS, and provide an example of the use of the objects defined in the previous section, we consider the calculation of the Jacobian, or sensitivity matrix, J.

Given a FEM model of an EIT medium, $F$, we calculate the vector of voltages, $\mathbf{v}$, for each FEM degree of freedom (mainly, degrees of freedom are nodes, but in the Complete Electrode Model [6], electrodes are also associated with a degree of freedom):

$$
\mathbf{v}=F(\sigma, \mathbf{q})
$$

where $\sigma$ is the vector of element conductivities and $\mathbf{q}$ is the current stimulation pattern, a vector of current inputs to each FEM degree of freedom. The measured electrode voltage can be represented as a linear combination of voltages, $\mathbf{V}_{t o E}$. For each simulation pattern, $\mathbf{q}_{i}$, a vector of measurements $\mathbf{m}_{i}$ are acquired, each of which consist of a linear combination of electrode measurements, represented by $\mathbf{M}_{i}$. Thus:

$$
\mathbf{m}_{i}=\mathbf{M}_{i} \mathbf{V}_{t o E} F\left(\sigma, \mathbf{q}_{i}\right)
$$

Based on this model, $\mathbf{J}$ is calculated as:

$$
\mathbf{J}_{i, j}=\left.\frac{\mathbf{M}_{i} \mathbf{V}_{t o E} \partial F\left(\sigma, \mathbf{q}_{i}\right)}{\partial \sigma_{j}}\right|_{\sigma=\sigma_{0}}
$$

where $\sigma_{0}$ is the "background" conductivity around which small changes are assumed to occur. To represent $\mathbf{J}$ as a matrix, measurements for all stimulation patterns $i$ are flattened into column vectors which are concatenated for each finite element $j$. In 


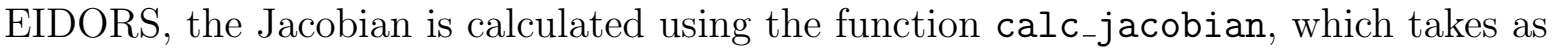
parameters the FEM model (type fwd_model) and the image of $\sigma_{0}$ (type image).

Objects may be created using the eidors_obj() function, which can fill in default attributes, and keeps track of cached properties. For example, given a $\mathrm{f} w d$ model, fem, and a homogeneous background image of conductivity one, img_bkgnd, may be computed by:

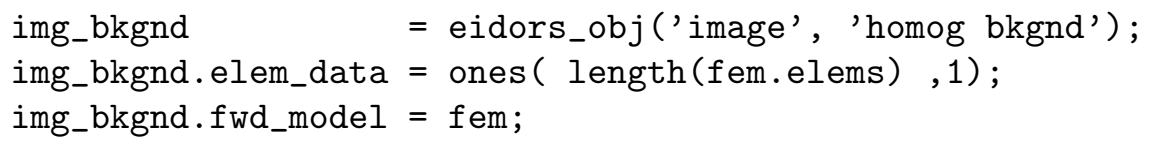

In this expression, the type field is assigned to 'image' and then name is assigned (arbitrarily) to 'homog bkgnd'.

In order to calculate the Jacobian, we call

$J=c_{-}$calcobian ( fem, img_bkgnd )

which, first, tests to see whether $\mathbf{J}$ has been previously calculated for $f$ em and img_bkgnd. If so, the cached value is returned; otherwise, it loads and calls the function in fem.jacobian, which may be np_calc_jacobian, which calls the function from the software of [11]; the returned value is then stored in the cache and returned to the calling function.

\section{Usage Examples}

In this section two examples of the usage of EIDORS software functions are provided. The first illustrates how a change in the electrode configuration and stimulation pattern can be done with a modification of the $\mathrm{fwd}$ model. The second illustrates how to modify the image reconstruction behaviour of an existing algorithm to add new features.

\subsection{Modification of electrode configuration}

In this section we wish to explore 3D EIT reconstructions using a sixteen electrode EIT system. This is a practical problem in many applications where a sixteen electrode EIT hardware system is available, and the goal is to choose where to put those electrodes to image a specific physiological process.

In our tests, we use a "zigzag" electrode placement using 16 electrodes, in which odd and even numbered electrodes are vertically separated onto two different planes (as illustrated in fig. 6). To illustrate the construction of this model, we begin with the demonstration finite element configuration given in EIDORS3D [11], which uses two vertically separated rings of 16 electrodes. applicable to this case.

In order to simulate data, a FEM model object mdl_3d and a conductivity image need to be created. EIDORS provides a utility function mk_common model to provide many of useful 2D and 3D models. This function may be used as follows to construct the FEM and use it to simulate voltage patterns. 


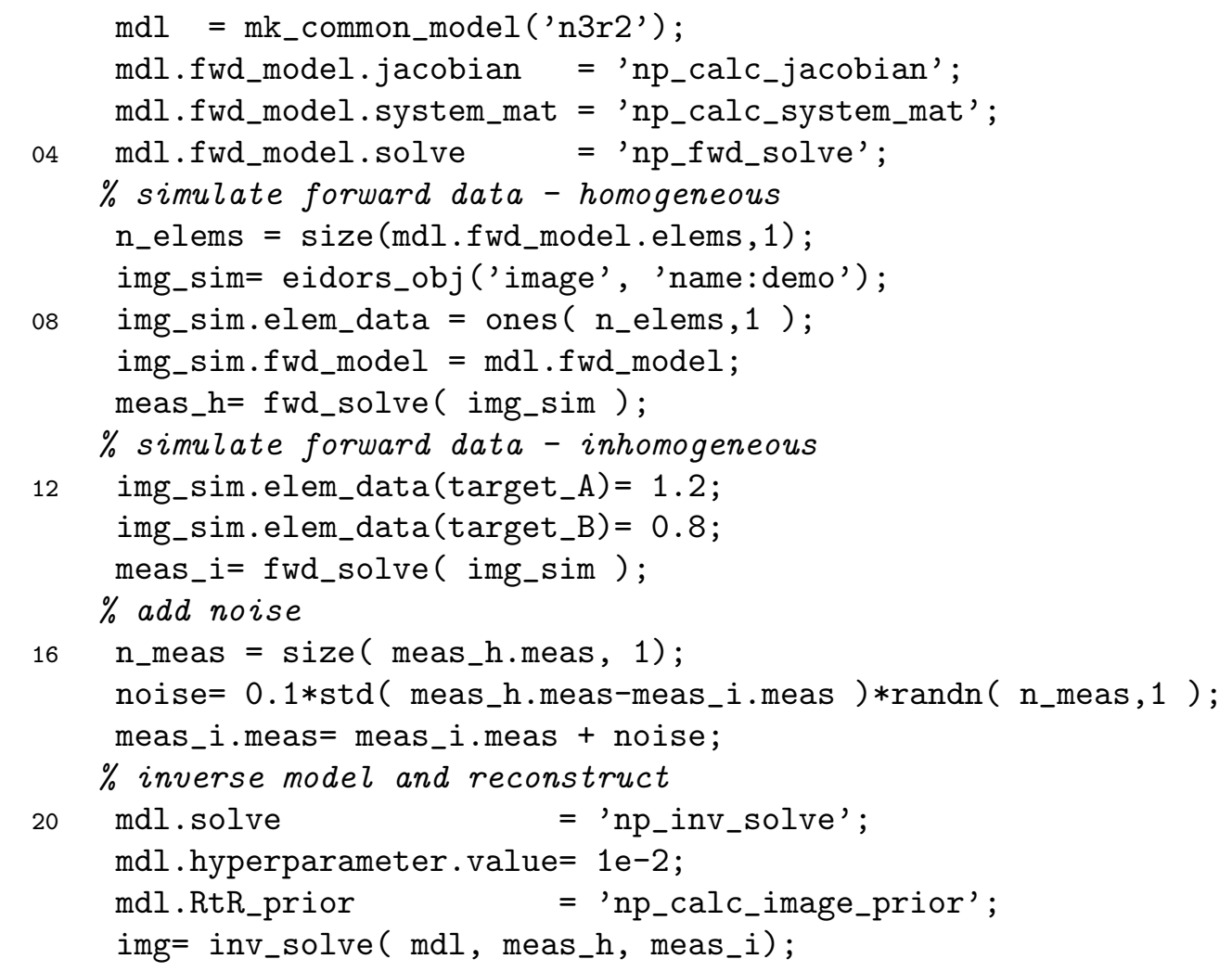

In this sample code, lines 1-4 set up the model and assign the functions to solve the forward problem. Lines 6-9 create an image object with a homogeneous conductivity in all elements, and solve the forward problem to simulate data, meas $\mathrm{h}$, on line 10 . To simulate an inhomogeneous model, lines 12-13 modify the conductivity distribution, and calculate meas_i on line 14, to which we add noise of signal to noise ratio (SNR) $20 \mathrm{~dB}$ on lines $16-18$. target_A and target_B are the indices of the elements associated with a non-conductive and conductive target shape. To solve the inverse problem, we assign the functions to the inverse model on lines 20-23, and call the inverse solver on line 24 to calculate the image img. This image may be displayed using the following imaging functions (Fig. 5):

24

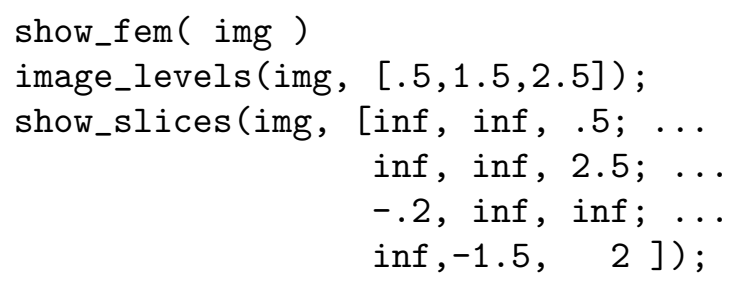

where show_fem images a 3D visualization of the FEM and image, image_levels displays slices through the $z$-axis and show_slices displays an arbitrary set of planar slices though the model, for which the $x-, y-$ and $z-$ axes are specified.

The functions illustrated build cylindrical EIT FEM models. In order to use more sophisticated simulations, these functions can be replaced by the user. For example, code to build models using netgen [15] is available in the meshing/netgen/ directory. In addition to the Matlab based visualization functions, functions are provided to write data to the VTK in the graphics_vtk directory, formatted to allow visualization using 

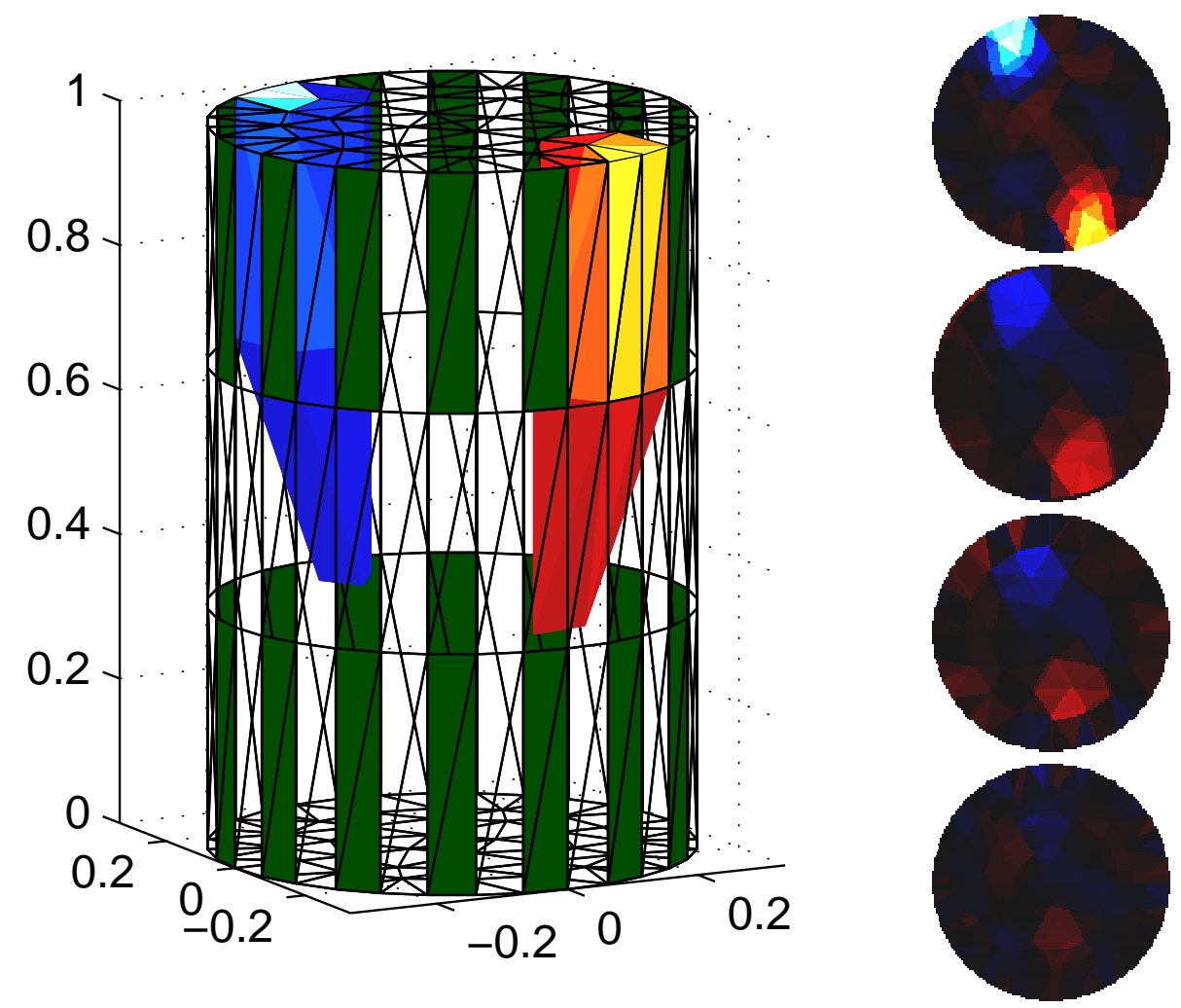

Figure 5. The 3D demonstration FEM provided with EIDORS3D [11], using two rings of 16 electrodes. Simulated data are used with added noise to $20 \mathrm{~dB}$ SNR. Left: FEM Model with coloured elements for reconstructed image values with an absolute value above 0.25 of the absolute maximum image value. Right: $2 \mathrm{D}$ slices through the reconstructed image at $z$-axis intercepts of $0.125,0.375$, 0.625 , and 0.875 from bottom to top.

Mayavi [13].

In order to use this model with a sixteen electrode EIT system, we need to: 1) modify and reorder the electrode definitions, and 2) define a new stimulation and measurement protocol. We implement this as follows:

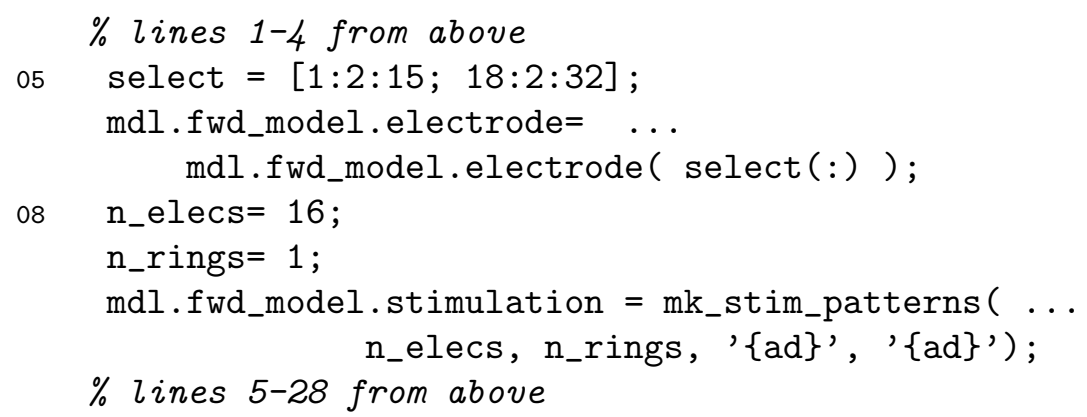

This modification of the model requires 7 lines to be added to the previous example. Lines 5-7 modify and reorder the electrodes, and lines 8-11 introduce a new, adjacent stimulation protocol with one ring of 16 electrodes. Reconstructed images are shown in 

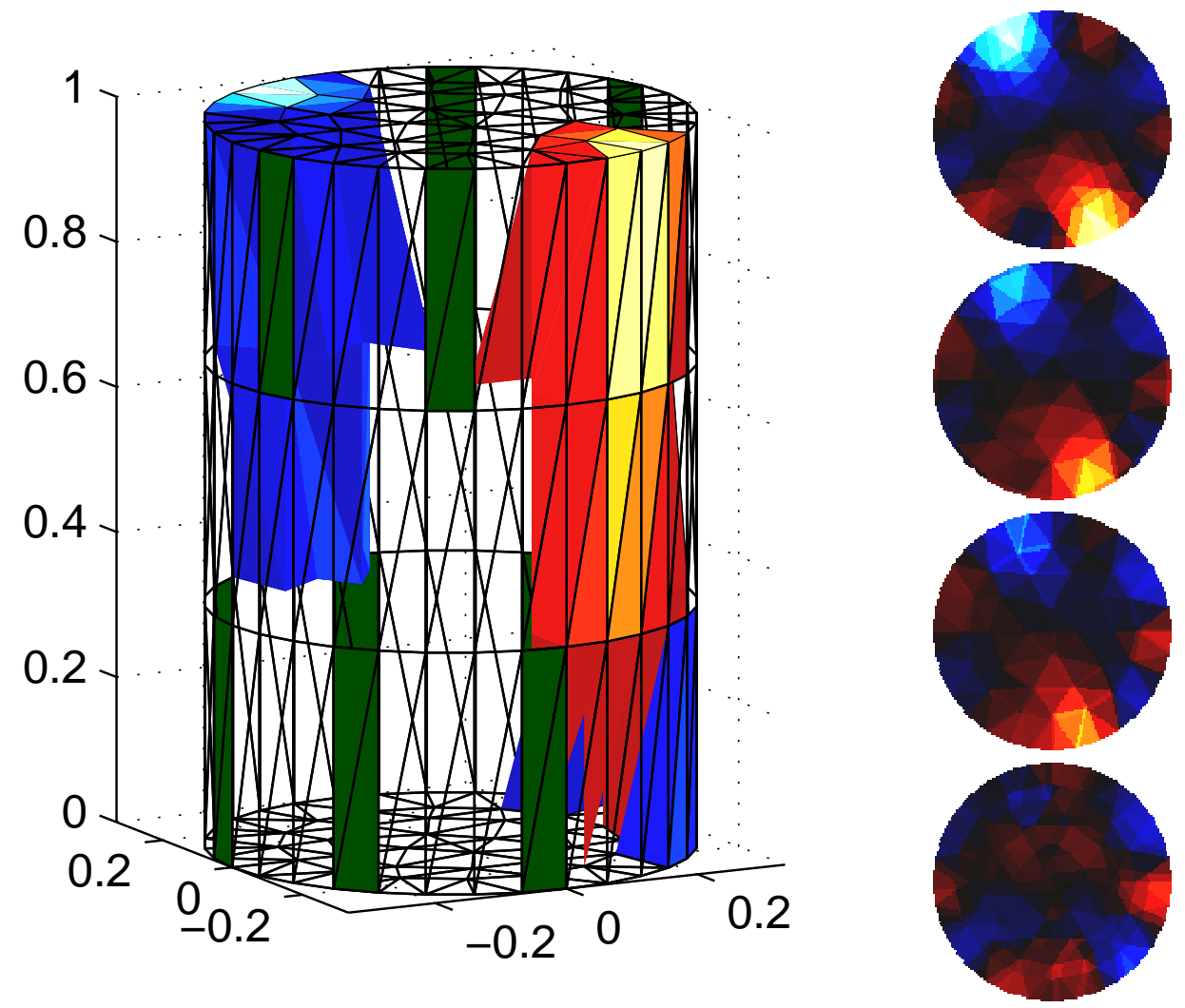

Figure 6. Images reconstructed using a modification of the model of 5 using 16 electrodes in a zigzag configuration. Electrodes are placed in a projected circle, but even and odd numbered electrodes are moved away from the plane in different directions in the $z$-axis. Simulated data are used with 20dB SNR AWGN added. Left: FEM Model with coloured and filled in elements for reconstructed image values what an absolute value above 0.25 of the absolute maximum image value. Right: $2 \mathrm{D}$ slices through the reconstructed image at $z$-axis intercepts of $0.125,0.375,0.625$, and 0.875 from bottom to top.

Fig. 6. It is clear that the image quality is lower in Fig. 6 than for Fig. 5; this is to be expected; this configuration has less than $\frac{1}{4}$ the number of independent measurements.

\subsection{Reconstruct Images}

This section shows how to use EIDORS to conveniently override the image prior for an existing algorithm. In the subsequent section, we discuss the importance of using correct image priors, and how image artefacts may easily be introduced by the incorrect choice of these parameters. The "plugability" feature of the EIDORS design allows this to be implemented in an convenient way.

In this section, we modify the example of the previous section to use an image prior defined by the function my_cheating_prior(); this function is similar to the prior of [12] but modifies it to reduce by half the reconstruction penalty at specific image 
elements chosen by the user. To do this, we replace line 22 from the previous example.

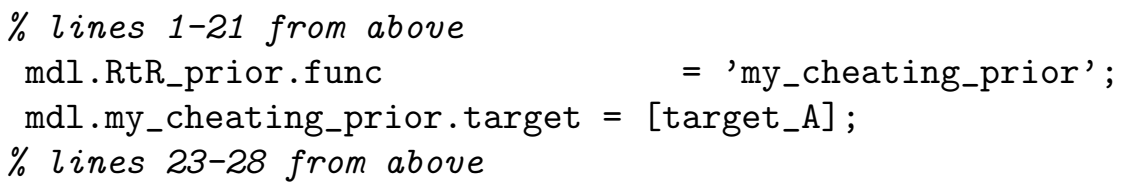

We define the function my_cheating_prior in a separate file:

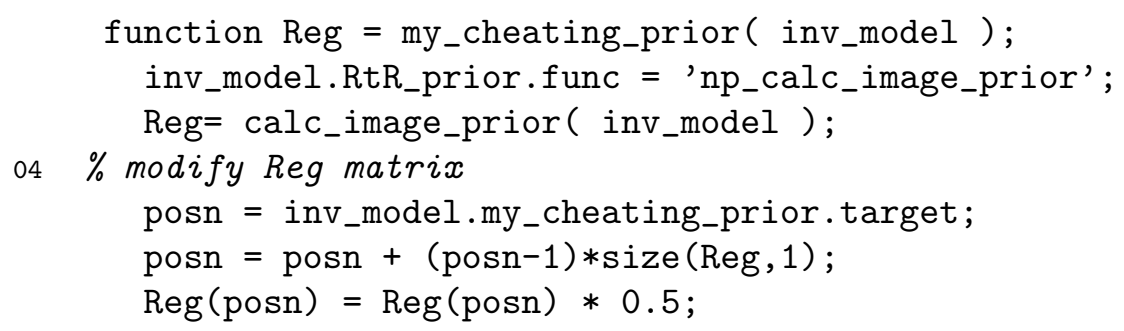

In lines $2-3$, the previous prior function is calculated from np_calc_image_prior(). Subsequently, on lines 5-7, the position of target elements is calculated and the penalty term multiplied by 0.5 . Line 6 calculates a column major index into the matrix Reg of the diagonal element indicated by posn. As expected, images reconstructed with this prior show sharper images than that of 6 . The implications of this type of "cheating" are explored in detail in the following section.

\section{Cheating with EIT}

One general issue with inverse problem software is the difficulty of correctly implementing algorithms, and the consequent ease with which subtle numerical bugs can be inadvertently introduced. EIDORS helps with this issue, by allowing sharing and reuse of well documented and debugged software. On the other hand, since EIDORS is designed to facilitate use by non-specialists, it may inadvertently encourage numerical errors. In order to address this issue, we develop in this section a list of ways in which such errors, or "cheats" may occur. Our hope is that such a list will assist authors of software to avoid such implemention issues.

\subsection{Sample Problem: The "happy" transform}

In this section, we describe some simple implementation errors that may occur with common, linear regularization algorithms. As the solution strategy becomes more complex, such as for non-linear and iterative imaging algorithms, there are more complex interactions between different parts of the algorithms, leading to more possibilities for "cheating".

To motivate the problem, assume that EIT measurement data have been acquired from a 2D simulated phantom which resembles a sad face. Being of an optimistic outlook, we wish that the reconstructed images represent a happy face instead. Fig. 7 illustrates what we will refer to as the "happy transform". We choose this example because it is important to distinguish two types of reconstruction artefacts. Most artefacts result in adding noise to images. While such noise is inconvenient, it does not cause a strong 
possibility of a misinterpretation. The most likely scenario is that an observer will conclude that there is an error, and ignore the results, or redo the measurements. On the other hand, some reconstruction artefacts can produce structured output which could result in a misinterpretation of the image. Such an error could result, in a medical imaging application, in a dramatically incorrect diagnosis. We have designed the "happy transform" to illustrate this type of image artefact.

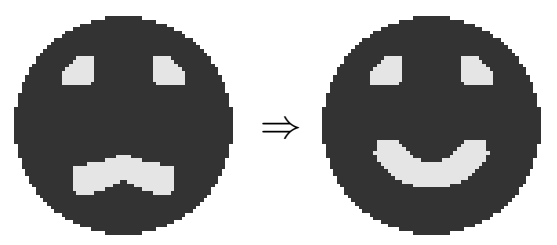

Figure 7. The happy transform. Left, a 2D phantom representing a sad face, and, right, a 2D phantom representing a happy face. The happy transform describes an image reconstruction method which (incorrectly) reconstructs data from the sad face phantom such that the image resembles the happy face.

\subsection{Cheating approach \#1: Careful selection of noise}

Typically, a reconstruction algorithm is presented with additive white Gaussian noise (AWGN) added to simulation data. One possible way to create the happy transform is to carefully select the noise introduced. For this example, we simulated homogeneous $\left(\mathbf{v}_{h}\right)$ and inhomogeneous $\left(\mathbf{v}_{i}\right)$ data on a 256 element FEM. Subsequently $17.75 \mathrm{~dB}$ of AWGN was added to $\mathbf{v}_{i}$, and the images reconstructed using the algorithm of [1]. Each image was reconstructed, and evaluated by us to detect which cases produced the "happy face". Examples are shown in Fig. 8.
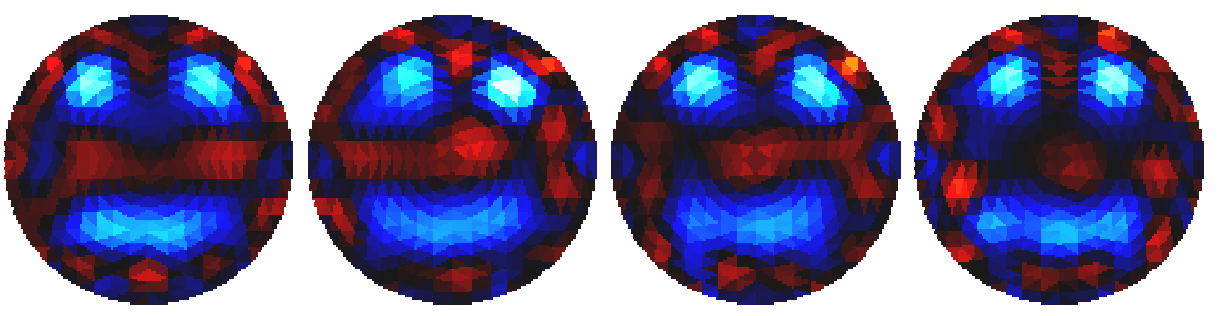

Figure 8. Images of data from figure 7 (left) with 17.75dB AWGN. Images were reviewed and selected if the output was visually "happy". Sample images are shown.

In order to determine the frequency with which AWGN noise may result in "happy" faces, 2000 images were reviewed and 41 were identified, corresponding to an occurrence rate of approximately $2 \%$. 


\subsection{Cheating approach \#2: Careful selection of priors}

Regularization techniques provide a powerful set of tools to approach ill-conditioned and ill-posed problems like EIT. At the same time, however, the use of a regularized algorithm implicitly makes probabilistic assumptions about the image element values and correlations. This section reviews ways in which incorrect assumptions for the prior probabilities may introduce artefacts into the reconstructed images.

We model linearized one-step difference EIT regularized image reconstruction by:

$$
\hat{\mathbf{x}}=\left(\mathbf{J}^{t} \mathbf{J}+\lambda^{2} \mathbf{R}\right)^{-1} \mathbf{J}^{t} \mathbf{y}
$$

where the $\hat{\mathbf{x}}$ is the reconstructed image, $\mathbf{y}$ is the vector of difference data, $\mathbf{J}$ is the FEM Jacobian, and $\mathbf{R}$ is the regularization matrix. Many difference EIT reconstruction algorithms have been formulated this way, such as [5], [1], and [12]. The Bayesian framework for regularization interprets the regularization matrix $(\mathbf{R})$ as $a$ priori information about the underlying image probability distribution; $\mathbf{R}$ is a penalty term which discourages reconstructed images with high values of $\hat{\mathbf{x}}^{t} \mathbf{R} \hat{\mathbf{x}}$. In practice, however, $\mathbf{R}$ is selected using ad hoc or heuristic techniques. If the prior does not model the actual data, then the reconstructed image will be biased.

In a Tikhonov regularization scheme, the amplitude of image elements is penalized. To illustrate this regularization scheme, we use a uniform weighting for each image element, using $\mathbf{R}=\mathbf{I}$.

If, however, it were known (or assumed) a priori that data were measured from a happy face, then we would not wish to penalize image elements which we know (or assume) to be large. Thus for each image element $i$ in the happy face, we set

$$
\mathbf{R}_{i, i}=0.5
$$

while all remaining diagonal elements of $\mathbf{R}$ are 1.0 .

The effect of modified Tikhonov prior selection is shown in Fig. 9A. In this case, images were reconstructed on a 576 element FEM (chosen to differ from the 256 element simulation mesh).

In order to enhance this effect, we use an inverse crime, which, as defined by [7], occurs when using the same model for both data simulation and image reconstruction. In this case, the regularized prior information is placed exactly at the positions of the happy face finite elements (Fig. 9B). The results show sharper region boundaries and a more pronounced 'happy transform' effect.

\subsection{Cheating approach \#3: Edge based priors}

Tikhonov priors impose a penalty on the amplitude of image elements, but do not model inter-element correlations. However, it is reasonable to assume, for most EIT applications, the conductivity distribution in the medium is largely smooth. It is thus advantageous to model this smoothness as a penalty for non-smooth image regions, using a matrix $\mathbf{R}$ based on an edge sensitive filter. One common edge sensitive filter is 


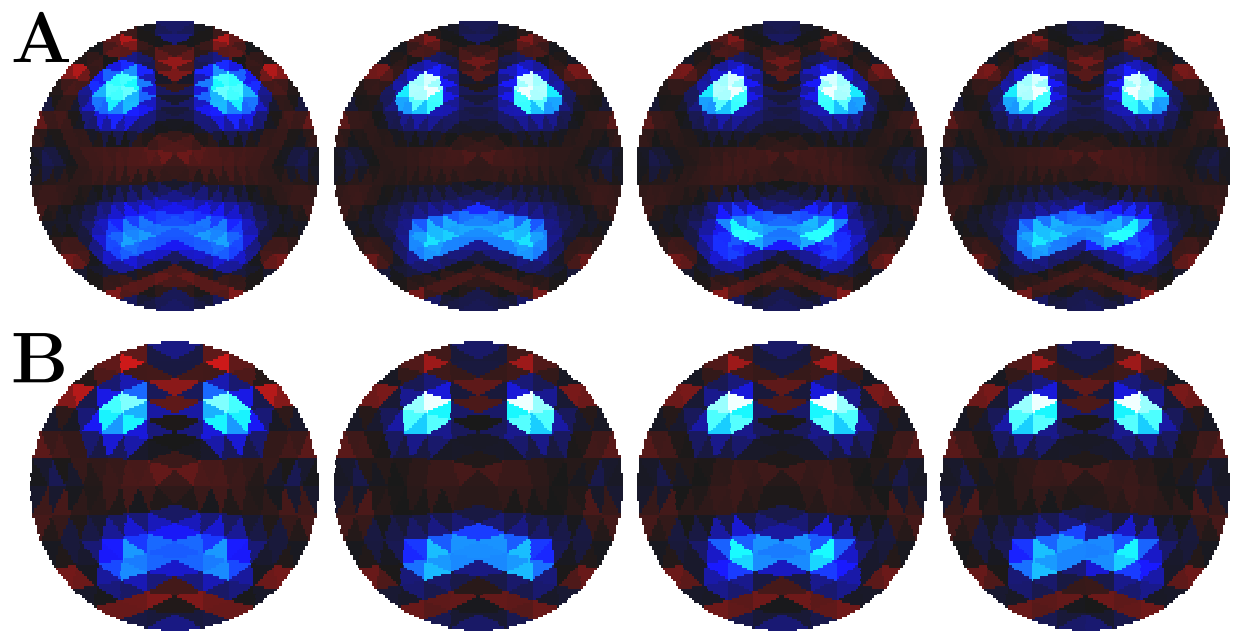

Figure 9. Reconstructed images illustrating the effect of modifications to Tikhonov image priors, using: Row $A$ a different mesh for model and reconstruction. Row $B$ the same mesh for model and reconstruction (ieinverse crime). Images are numbered from left to right. Image 1: Tikhonov prior with no weighting, Image 2: Tikhonov prior with weighting for positions in sad face, Image 3: Tikhonov prior with weighting for positions in happy face, Image 4: Tikhonov prior with weighting for sad face (left) and happy face (right),

the discrete Laplacian, in which the penalty term is calculated for each element and its surrounding finite element neighbors. Each neighboring element is assigned a weighting of -1 and the element itself has a weighting of $D+1$, where $D$ is the model dimension, in order for the penalty term to sum to zero for smooth images. Thus,

$$
R_{i, j}= \begin{cases}D+1 & \text { if } i=j \\ -1 & \text { if element } i \text { is adjacent to } j \\ 0 & \text { otherwise. }\end{cases}
$$

Such a Laplacian filter can be used as a regularization prior to penalize high frequency components (edges) in the image. In a similar way to the Tikhonov case, if we know (or assume) where the edges are, then edges should not be penalized (or be less penalized) in those places. Fig. 10A shows the effect of such careful edge preserving prior selection (with no inverse crime). Known edges are weighted at 0.2 times that of other edges in the image. Again, the inverse crime produces sharper image contrasts and a more pronounced effect. In order to enhance this effect, we use an inverse crime, by putting the prior information exactly where edges occur in the happy face (Fig. 10)B. Known (or assumed) edges are weighted at 0.2 times that of other edges in the image.

\subsection{Cheating approach \#3: Reconstruction of Three fingers}

We now illustrate some cheating for a three dimensional problem. We model a cylindrical phantom tank with a rather coarse FEM mesh of only 828 elements with 32 rectangular 

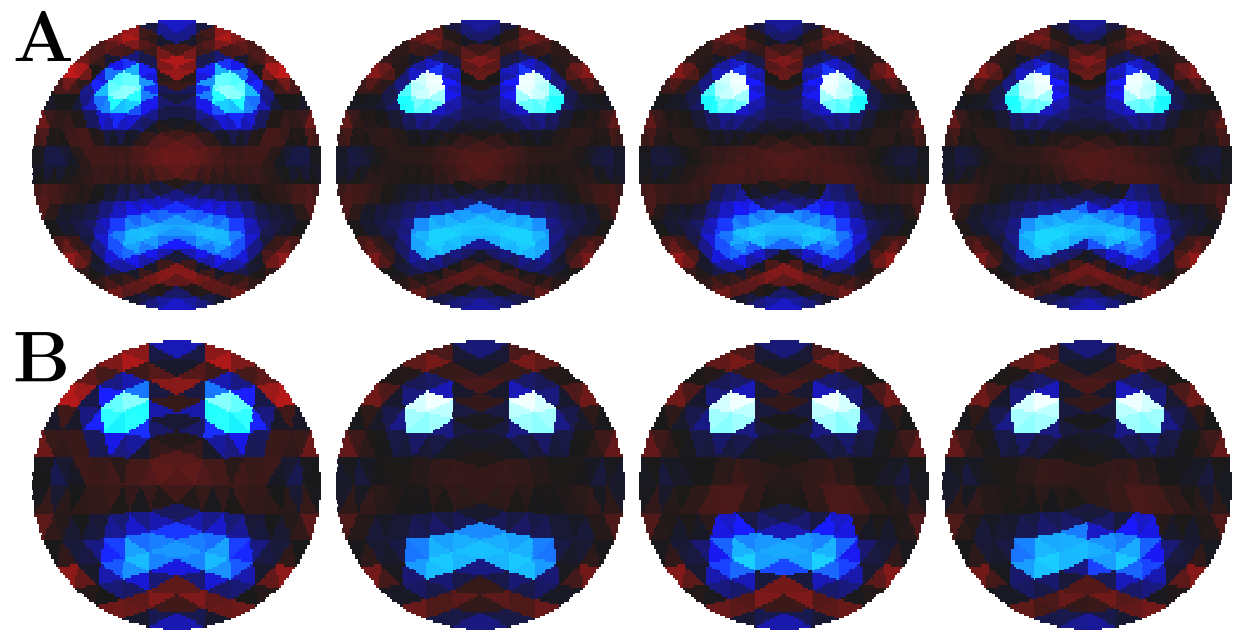

Figure 10. Reconstructed images illustrating the effect modifying the weighting of edge preserving image priors, using: Row $A$ a different mesh for model and reconstruction. Row $B$ the same mesh for model and reconstruction (ieinverse crime). Images are numbered from left to right. Image 1: Edge prior with no weighting, Image 2: Edge prior with weighting for positions in sad face, Image 3: Edge prior with weighting for positions in happy face, Image 4: Edge prior with weighting for sad face (left) and happy face (right),
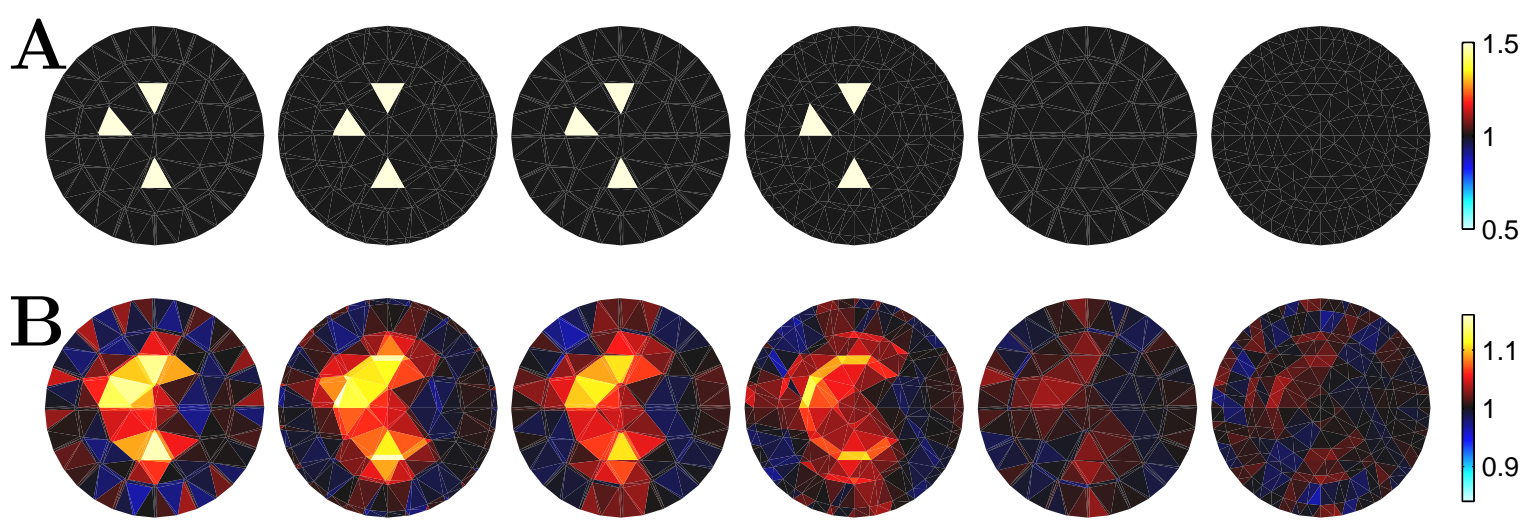

Figure 11. Row $A$ : The three fingers test object cross section used to simulate voltage data. Row B: Initial reconstruction of the three finger object. From left to right, cross sections of reconstructed volume at $z$-axis values of $0.1,0.83,1.1,1.72,2.1$, and 2.63. Colour mapping is uniform for each row (colourbar at right).

electrodes in two planes. Note that to realistically fit data even for a homogeneous tank to a sensible measurement accuracy requires a much finer mesh, but the principles of cheating will be similar. The mesh geometry was constructed using constructive 
solid geometry in netgen. The electrodes were created by the intersection of cuboids with the cylinder representing the tank. The target object is three triangular prisms projecting from the base of cylinder as a crude approximation to three fingers inserted in a saline filled tank. To make the simulated data, we used a mesh with added contrasts to model these fingers (Fig. 11 Left) and added Gaussian pseudo-random error to this data with a variance of $10^{-5}$ of the maximum voltage. The images shown are typical of those generated from data from several draws of the pseudo random number generator. We then attempt to reconstruct the conductivity using a single step of regularized Gauss-Newton. Note that we do not expect to do very well with a crude mesh and reconstruction algorithm, but we are interested to see if we can distinguish three fingers and their approximate position. Our first attempt, using an arbitrarily chosen regularization factor of $10^{-4}$ appears in Fig. 12A. It is displayed using the VTK visualization program MayaVi [13], after exporting the data to a vtk file using the EIDORS function writevtkfile(filename, model, image). The piecewise constant conductivity of the reconstruction has been interpolated using the CellToPointData filter in Mayavi, and displayed using Mayavi's isosurface with the contour level selected manually. The image projection has been selected to best see the fingers.

Cross sectional images of original and reconstructed conductivity are shown in Fig. 11. The contour selected for display is itself a hyperparameter and its choice is susceptible to cheating. In the absence of a priori information about the number or shape of the target some automatic selection procedure is needed. Mayavi's default contour selection in this case produces an image that is not as easy to interpret as three fingers (Fig. 12B).
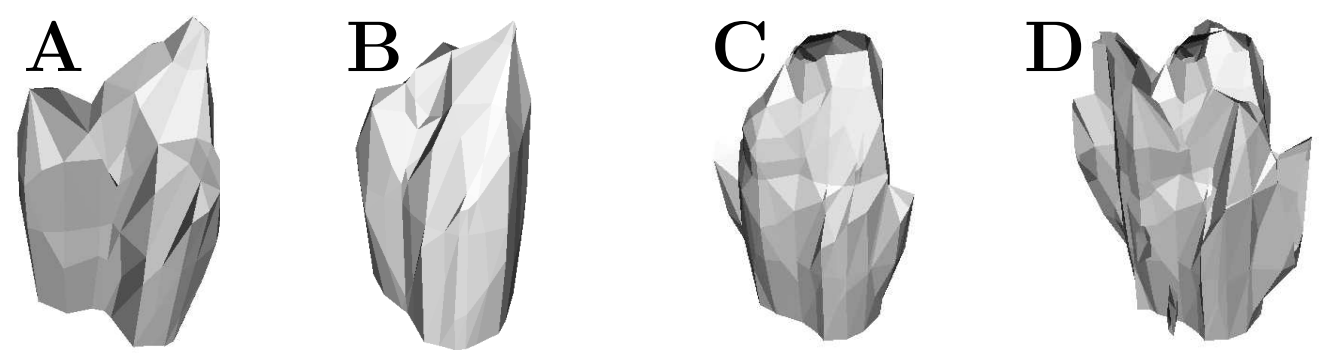

Figure 12. $A$ : Initial reconstruction of the three finger object $B$ : Mayavi's automatic selection of contour $C$ : The effect of decreasing the Tikhonov regularization parameter by a factor of $10 \mathrm{D}$ : Seemingly different number of fingers resulting simply from a different choice of contour

Choosing a smaller regularization parameter will of course generate a less smooth conductivity and we might easily interpret such an image as having a different number of fingers. Fig. 12C illustrates the effect of reducing the regularization parameter by a factor of 10 . Similarly an unfortunate choice of contour could produce an image susceptible to interpreting more fingers (Fig. 12D). 


\section{Discussion}

This paper presents version 3.1 of the EIDORS open source software suite for image reconstruction in electrical impedance and diffuse optical tomography. The current version builds on the capabilities of earlier versions of EIDORS [17], [11], but has a different focus. Rather than simply providing image reconstruction software, EIDORS version 3 takes a software engineering approach to its design, in order to address the following practical issues.

- Extensible software: In order to see community use and participation in EIDORS, it is important to provide a software framework which makes it clear how the pieces are used and fit together. We address this issue by providing many simple and more detailed examples, and by implementing a unified "pluggable" software architecture, into which multiple FEM models, image algorithms, and interfaces to common hardware are provided.

- Design for testability: In our experience, correct design of software for inverse problems such as EIT is difficult. Among the many causes we identify: 1) the numerical issues associated with the solution of ill-conditioned problems on large linear algebra systems, and 2) the ease with which subtle model errors can cause image artefacts. In the EIDORS software, we attempt to assist users of the software as follows: For numerical difficulties, we provide several well used independent algorithms to solve core functions, as well as a test suite to validate the calculations of several important parameters. The algorithm model errors, on the other hand, we are not able to address in software. Instead, we have written a section on how to identify and avoid such errors as part of this paper. We hope that these ideas will help clarify the kinds of possible errors, and help researchers to avoid them.

We make the following additional observations related to "cheating" with inverse problems. We intentionally take a lighthearted tone because we do not want to imply that anyone is being accused of making such errors. Furthermore, in our experience, it is surprisingly easy to unwittingly develop algorithms which are subject to variants of these cheats. As mentioned, many algorithm errors simply result in noisy artefacts in the images. These errors are not a big concern, in the sense that their presence is obvious. Such cases can be described by the aphorism: "Garbage in; garbage out". The worrisome errors are those that introduce the possibility of false image interpretation, since the quality of the produced image does not obviously call it into question. The example of a transformation of a sad to happy face, or the calculation of the wrong number of fingers, was chosen to illustrate this kind of error. It would clearly be preferable for an algorithm to produce an obviously wrong output - noise - rather than one that can be misinterpreted in this way. We would therefore like to introduce the following edict: "Don't whitewash our garbage!".

In conclusion, we have described the new developments of the EIDORS software for the version 3.1 release. These developments have been focussed on software engineering 
improvements that will facilitate collaborative use, modification and enhancement of the software by the EIT community. Open source software like EIDORS could potentially be a dramatical help to research and development in medical imaging fields like EIT. We are hopeful that EIDORS will assist in the development and testing of new approaches and ideas, and will ease the use of advanced algorithms in experimental, industrial and clinical applications of these technologies.

\section{References}

[1] Adler A and Guardo R 1996 Electrical impedance tomography: regularised imaging and contrast detection IEEE Trans. Med. Imaging 15 170-9

[2] Asfaw Y and Adler A 2005 Automatic detection of detached and erroneous electrodes in electrical impedance tomography Physiol. Meas., 26 S175-S183

[3] Borsic A 2002 Regularisation Methods for Imaging from Electrical Measurements PhD thesis, Oxford Brookes University, U.K.,

[4] Cederqvist P 2002 Version Management with CVS, Network Theory Ltd, Bristol, UK

[5] Cheney M Isaacson D Newell J C Simske C and Goble J C 1990 NOSER: An algorithm for solving the inverse conductivity problem Int. J. Imaging Systems \& Technol. 2 66-75

[6] Cheng K-S Isaacson D Newell J C and Gisser D G 1989 Electrode models for electric current computed tomography IEEE Trans Biomed Eng 36 918-924

[7] Colton D and Kress R 1992 Inverse Acoustic and Electromagnetic Scattering Theory Springer, Berlin.

[8] Eaton J W 2002 Gnu Octave Manual Network Theory Ltd, Bristol, UK

[9] Free Software Foundation 1991 GNU General Public Licence Boston MA USA http://www.gnu.org/copyleft/gpl.html

[10] Gamma E Helm R Johnson R and Vlissides J 1995 Design Patterns: Elements of Reusable ObjectOriented Software Addison-Wesley, Boston, MA, USA

[11] Polydorides N and Lionheart W R B 2002 A Matlab toolkit for three-dimensional electrical impedance tomography: a contribution to the Electrical Impedance and Diffuse Optical Reconstruction Software project, Meas. Sci. Technol. 13 1871-1883

[12] Polydorides N 2002 Image reconstruction algorithms for soft-field tomography Ph.D. Thesis, University of Manchester Institute of Science and Technology, U.K.

[13] Ramachandran P 2003 Scientific data visualization with MayaVi Conf. SciPy: Python for Scientific Computing Pasadena, CA, USA http://mayavi.sf.net/

[14] Soleimani M Gómez-Laberge C and Adler A 2005 Imaging of conductivity changes and electrode movement in EIT Physiol. Meas., submitted

[15] Schöberl J 1997 NETGEN - An advancing front 2D/3D-mesh generator based on abstract rules. Comput. Visual. Sci 1 41-52 http://www.hpfem.jku.at/netgen/

[16] Vauhkonen M 1997 Electrical impedance tomography and prior information $\mathrm{PhD}$ thesis, University of Kuopio, Finland

[17] Vauhkonen M, Lionheart W R B, Heikkinen L M, Vauhkonen P J and Kaipio J P, 2000 A MATLAB package for the EIDORS project to reconstruct two-dimensional EIT images Physiol. Meas. 22 $107-111$ 